\title{
Utilização de Modelos Autoregressivos na Quantificação de Incertezas em Problemas de Transporte Linear ${ }^{\dagger}$
}

\author{
T. JORDEM PEREIRA ${ }^{1 *}$ e H.P. AMARAL SOUTO ${ }^{2}$
}

Recebido em 28 agosto, 2014 / Aceito em 9 fevereiro, 2016

\begin{abstract}
RESUMO. O fenômeno físico do processo de escoamentos de traçadores em um meio poroso heterogêneo é modelado por um sistema de equações diferenciais parciais, sujeitas a certas condições de contorno e inicial. As variações significativas das propriedades do meio poroso (porosidade e permeabilidade) são responsáveis pela introdução das incertezas contidas no modelo matemático. Com intuito de reduzir as incertezas dos modelos geológicos, deferentes metodologias tem sido desenvolvidas e testadas em diversos problemas de escoamentos de fluidos em meios porosos heterogêneo. O objetivo deste trabalho é o estudo da quantificação de incertezas em problemas de escoamentos de traçadores em meios porosos heterogêneos empregando uma abordagem Bayesiana para a seleção dos campos de permeabilidades, baseada em um conjunto de medições da concentração do traçador em pontos específicos do meio poroso. O método da Soma Sucessiva de Campos Gaussianos Independentes (SSCGI) é utilizado na parametrização das incertezas contidas nos meios porosos heterogêneos. Na resolução do problema inverso, emprega-se o método de Monte Carlo via Cadeias de Markov (MCMC) a dois estágios que utiliza o algoritmo de MetropolisHastings baseado em passeios aleatórios do tipo autoregressivo. Através deste procedimento, gera-se uma cadeia Markov que converge para a distribuição estacionária, que neste caso é a distribuição a posteriori de interesse. Resultados numéricos são apresentados para um conjunto de realizações dos campos de permeabilidades.
\end{abstract}

Palavras-chave: Campos estocásticos Gaussianos, escoamento em meios porosos, método MCMC a dois estágios, traçador.

\section{INTRODUÇÃO}

O estudo dos escoamentos multifásicos em meios porosos heterogêneos representa uma ferramenta útil em diversas áreas importantes para o desenvolvimento científico e tecnológico, dentre as quais pode-se destacar a indústria do petróleo e a contaminação ambiental. As técnicas

\footnotetext{
$\dagger$ Trabalho apresentado no XXXV Congresso Nacional de Matemática Aplicada e Computacional.

*Autor correspondente: Thiago Jordem Pereira.

${ }^{1}$ Departamento de Ciências Exatas, Biológicas e da Terra, INFES, UFF, 28470-000 Santo Antônio de Pádua, RJ, Brasil. E-mail: tjordem@gmail.com

2 Departamento de Modelagem Computacional, Instituto Politécnico, UERJ, 28601-970 Nova Friburgo, RJ, Brasil.

E-mail: helio@iprj.uerj.br
} 
que empregam a injeção de traçadores vêm se mostrando viáveis no estudo de problemas relacionados à simulação numérica de escoamentos miscíveis de fluidos incompressíveis em reservatórios de petróleo, bem como no transporte de contaminantes em reservatórios subterrâneos. O tempo de chegada e a concentração do traçador nos poços de produção ou aquíferos são informações importantes para se identificar barreiras ao escoamento, caminhos preferenciais do escoamento, falhas geológicas, propriedades do meio poroso, comunicação entre reservatórios, condutividade hidráulica, dispersividade, dentre outros, que são difíceis de se obter através de técnicas geofísicas convencionais [3, 15]. Exemplos de simulações numéricas e de aplicações desta técnica podem ser encontradas nos trabalhos de [1, 2, 4, 17, 18, 19, 22].

Matematicamente, o escoamento do traçador é modelado por um sistema de equações diferenciais parciais sujeito a certas condições de contorno e inicial. Este sistema de equações diferencias é constituído de um sub-sistema elíptico, que determina a pressão e a velocidade da mistura, e de uma equação de transporte, que determina em cada instante de tempo o valor da concentração do fluido injetado (traçador). A obtenção de soluções numéricas deste sistema de equações diferencias parciais não é uma tarefa fácil visto que elas são fortemente influenciadas pelas propriedades do meio poroso, tais como a porosidade e a permeabilidade, cuja caracterização precisa via a construção numérica de campos de porosidades e permeabilidades ainda é um problema complexo. Estas propriedades são responsáveis pela introdução das incertezas contidas no modelo matemático, que ocorrem em múltiplas escalas, e que reduzem de forma significativa a confiabilidade das previsões obtidas mediante a resolução destas equações. Desta forma, uma grande importância vem sendo dada à aplicação de diferentes tipos de metodologias capazes de quantificar e reduzir as incertezas dos modelos geológicos e, consequentemente, obter previsões acuradas para, por exemplo, a produção de óleo em reservatórios de petróleo e o monitoramento do transporte de contaminantes em formações subterrâneas [10, 13, 14, 21].

A metodologia Bayesiana [16] pode ser utilizada na quantificação de incertezas em problemas envolvendo escoamentos de fluidos em meios porosos heterogêneo [10, 13, 14, 21]. Nessa abordagem, utiliza-se o Teorema de Bayes para atualizar as informações do parâmetro de interesse (permeabilidade ou porosidade) do modelo geológico. O Teorema de Bayes é uma ferramenta que sintetiza duas fontes de informações a distribuição a priori e a distribuição amostral dos dados (expressa formalmente pela função de verosimilhança), obtendo-se a distribuição a posteriori do parâmetro de interesse $[14,16]$. Geralmente, as informações atualizadas do parâmetro de interesse descritas pela distribuição a posteriori podem ser resumidas em termos que envolvem a avaliação de esperanças condicionais. Devido à complexidade de muitos dos modelos probabilísticos, o cálculo das esperanças condicionais não podem ser resolvidas analiticamente. Desta forma, recorre-se a procedimentos numéricos acurados e apropriados para a resolução do problema em questão, visando a melhorar a ordem de aproximação das variáveis de interesse.

Os procedimentos numéricos baseados em simulações estocásticas têm sido bastante utilizados para explorar a complexa distribuição a posteriori, dentre os quais pode-se destacar o método MCMC a dois estágios, abreviatura do inglês Markov Chain Monte Carlo (Monte Carlo via Cadeias de Markov) $[12,13]$. Este método faz o uso de algoritmos específicos para gerar cadeias de Markov ergódicas cuja distribuição estacionária é a distribuição de probabilidades a posteriori do parâmetro de interesse. Neste trabalho, utiliza-se o algoritmo de Metropolis-Hastings base- 
ado em passeio aleatório random walk do tipo autoregressivo [16]. Este algoritmo baseia-se na técnica de Aceitação-Rejeição, onde amostras são geradas a partir de uma distribuição instrumental a aceitas ou rejeitadas com uma certa probabilidade. O método MCMC a dois estágios têm sido bastante apropriado para a exploração da complexa distribuição a posteriori do problema de escoamentos de fluidos em meios porosos heterogêneos $[12,13,14]$.

O objetivo deste trabalho é o estudo da quantificação de incertezas em problemas inversos envolvendo o transporte de um traçador em meios porosos heterogêneos, empregando uma abordagem Bayesiana para a atualização dos campos de permeabilidades.

\section{OS MODELOS DE ESCOAMENTO DO TRAÇADOR}

Nesta seção, apresenta-se os sistemas de equações diferenciais parciais usados na modelagem, nas escalas de laboratório (escala fina) e de campo (escala grossa), do escoamento de traçadores em meios porosos heterogêneos. Nestes modelos, supõe-se que a porosidade é igual a uma constante, o meio porosos é indeformável e está saturado por um fluido. Além disso, os efeitos da gravidade e da compressibilidade foram negligenciados. O transporte é puramente advectivo e ausente de fontes ou sorvedouros. Uma certa quantidade de um traçador é injetada no fluido que escoa no interior do meio poroso. A concentração total do traçador é baixa o suficiente de modo que o fluido marcado e o residente tenham, em termos práticos, as mesmas propriedades físicas.

\subsection{O modelo para a malha fina}

O modelo matemático na escala fina usado para descrever o processo de escoamento de traçadores em um meio poroso $\Omega \subseteq \mathbb{R}^{2}$, sobre um intervalo de tempo $I=[0, T] \subset \mathbb{R}, 0 \leq t \leq T$, é constituído por uma equação de transporte linear da concentração do fluido marcado

$$
\frac{\partial c}{\partial t}+\mathbf{u} \cdot \nabla c=0, \quad \text { em } \Omega \times[0, T],
$$

e o campo de velocidades que é determinado pela lei de Darcy juntamente com a hipótese de incompressibilidade do fluido

$$
\mathbf{u}=-\frac{k(\mathbf{x})}{\mu} \nabla p, \quad \nabla \cdot \mathbf{u}=0, \quad \text { em } \Omega \times[0, T],
$$

onde a variável $\mathbf{x}=(x, y)$ é o vetor posição, $k(\mathbf{x})=k$ é um campo escalar de permeabilidades, com distribuição log-normal, a ser especificado, $\mathbf{u}=\mathbf{u}(\mathbf{x}, t)$ é a velocidade do fluido ou velocidade de Darcy e $\mathbf{p}=\mathbf{p}(\mathbf{x}, t)$ é a pressão do fluido através do meio poroso. O coeficiente $\mu$ representa a viscosidade da mistura e $\mathbf{c}=\mathbf{c}(\mathbf{x}, t)$ a concentração do fluido marcado.

As Equações (2.1) e (2.2) são definidas em um domínio quadrangular $\Omega=\left[0, L_{x}\right] \times\left[0, L_{y}\right]$, com contorno regular $\partial \Omega$ e, um intervalo de tempo $0 \leq t \leq T$, com condições de contorno dadas por

$$
\begin{aligned}
\mathbf{u} \cdot \mathbf{n} & =-q, \quad \text { em } x=0 \\
p & =0, \quad \text { em } x=L_{x}, \\
\mathbf{u} \cdot \mathbf{n} & =0, \quad \text { em } y=0 \mathrm{e} L_{y},
\end{aligned}
$$


e condição inicial do tipo Riemann

$$
c(\mathbf{x}, 0)= \begin{cases}1, & \text { se } x \leq 0, \\ 0, & \text { se } x>0,\end{cases}
$$

onde $\mathbf{n}$ é o vetor normal unitário exterior a $\partial \Omega, L_{x}$ e $L_{y}$ representam, respectivamente, as dimensões física do meio poroso nas direções $x$ e $y$, conforme a Figura 1. A taxa de injeção do traçador é dada por $q, c(\mathbf{x}, 0)=1$ representa a concentração do fluido injetado (traçador) e $c(\mathbf{x}, 0)=0$ é a concentração do traçador no fluido residente.

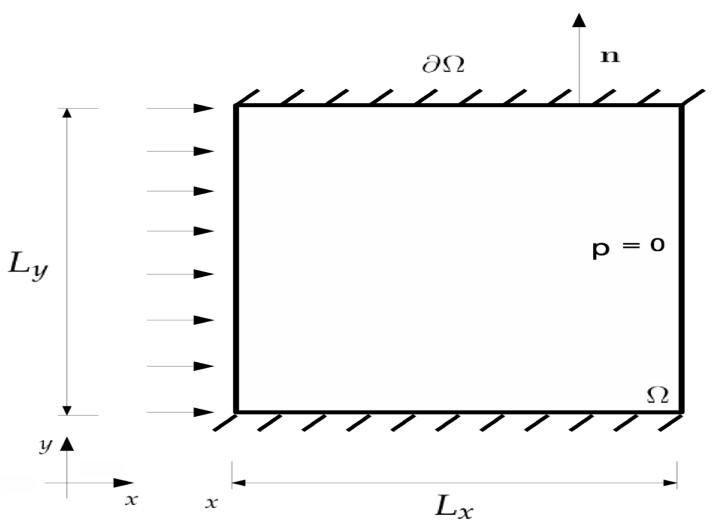

Figura 1: Domínio $\Omega$.

\subsection{O modelo para a malha grossa}

O método MCMC a dois estágios utiliza um modelo matemático na escala de campo (malha grossa) a fim de reduzir o esforço computacional e aumentar a taxa de aceitação das amostras propostas dos campos de permeabilidades $[13,14]$. Desta forma, uma técnica de upscaling do campo de permeabilidades se torna necessária de modo a obter-se valores de permeabilidades efetivas em cada elemento da malha grossa e as equações efetiva, apropriadas para a simulação numérica na escala de campo do problema do escoamento do traçador no interior do meio poroso $[13,14]$. A Figura 2 representa esquematicamente as malhas nas escalas fina e grossa.

Para calcular-se as permeabilidades efetivas na malha grossa, resolve-se para cada elemento $\Omega_{\alpha}$ da malha grossa o seguinte problema local:

$$
\begin{aligned}
\nabla \cdot\left[k(\mathbf{x}) \nabla \zeta_{i}(\mathbf{x})\right] & =0, & & \text { em } \Omega_{\alpha}, \\
\zeta_{i}(\mathbf{x}) & =\hat{g}_{i}(\mathbf{x}), & & \text { sobre } \partial \Omega_{\alpha},
\end{aligned}
$$

onde $g_{i}(\mathbf{x})$ são funções definidas no contorno do elemento da malha grossa $\Omega_{\alpha}$. Para que a solução deste problema seja obtida, é necessário estabelecer as seguintes condições de contorno:

$$
\begin{aligned}
\zeta_{i}(\mathbf{x}) & =1, \quad \text { em um lado do elemento na direção } \mathbf{e}_{i}, \\
\zeta_{i}(\mathbf{x}) & =0, \quad \text { no lado oposto do elemento na direção } \mathbf{e}_{i}, \\
\nabla \zeta_{i}(\mathbf{x}) \cdot \mathbf{n} & =0, \quad \text { fluxo nulo nos outros lados do elemento, }
\end{aligned}
$$




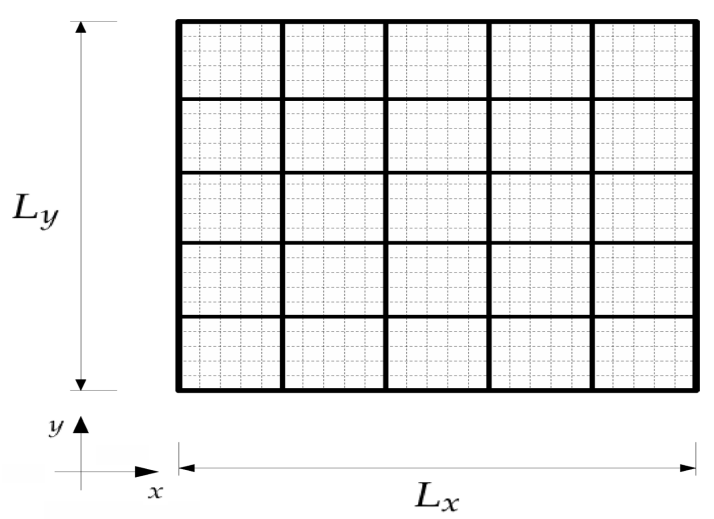

Figura 2: Representação esquemática das malhas nas escalas fina (linhas tracejadas) e grossa (linhas contínuas).

onde $\mathbf{e}_{i}$ é o vetor da base canônica. Assim, o tensor efetivo de permeabilidades é dado por:

$$
\left(\bar{k}(\mathbf{x}) \mathbf{e}_{i}, \mathbf{e}_{j}\right)=\frac{1}{\left|\Omega_{\alpha}\right|} \int_{\Omega_{\alpha}}\left(k(\mathbf{x}) \nabla \zeta_{i}(\mathbf{x}), \mathbf{e}_{j}\right) d \mathbf{x},
$$

onde $\zeta_{i}(\mathbf{x})$ é a solução da Equação (2.5) para as condições de contorno (2.6) e (a, b) representa o produto interno entre os vetores a e b.

Uma vez determinado o tensor efetivo de permeabilidades, o modelo matemático na malha grossa é constituído por

$$
\begin{gathered}
\frac{\partial \bar{c}}{\partial t}+\overline{\mathbf{u}} \cdot \nabla \bar{c}=0, \quad \text { em } \Omega \times[0, T], \\
\overline{\mathbf{u}}=-\frac{\bar{k}(\mathbf{x})}{\mu} \nabla \bar{p}, \quad \nabla \cdot \overline{\mathbf{u}}=0, \quad \text { em } \Omega \times[0, T],
\end{gathered}
$$

onde $\bar{c}=\bar{c}(\mathbf{x}, t), \bar{u}=\bar{u}(\mathbf{x}, t), \bar{p}=\bar{p}(\mathbf{x}, t)$, indicam, respectivamente, a concentração do traçador, a velocidade e a pressão do fluido na malha grossa. As Equações (2.7) e (2.8) estão sujeitas às condições de contorno e inicial análogas às definidas em (2.3) e (2.4).

\section{MODELAGEM ESTOCÁSTICA DA GEOLOGIA}

Para a parametrização das incertezas contidas nos meios porosos heterogêneos, emprega-se campos de permeabilidades escalares $k(\mathbf{x})$ com distribuição log-normal da forma:

$$
k(\mathbf{x})=k_{0} e^{\varphi Y(\mathbf{x})},
$$

onde $k_{0}$ é uma constante, $\varphi$ é o fator que controla o grau de heterogeneidade do meio poroso e $Y(\mathbf{x})$ é um campo aleatório Gaussiano caracterizado por sua média $\langle Y(\mathbf{x})\rangle=0$ e sua função de covariância dada por uma lei de potência [5]

$$
C(\mathbf{x}, \mathbf{y})=\langle Y(\mathbf{x}) Y(\mathbf{y})\rangle=|\mathbf{x}-\mathbf{y}|^{-\beta}, \quad \beta>0,
$$


onde \langle\rangle indica a média do conjunto e $\beta$ é o coeficiente de Hurst.

Gera-se os campos aleatórios $Y(\mathbf{x})$ utilizando o método das Soma Sucessiva de Campos Gaussianos Independentes (SSCGI) [5]. Este eficiente procedimento gera, numericamente, campos aleatórios Gaussianos correlacionados, baseando-se na superposição hierárquica de malhas uniformes independentes, isto é,

$$
Y(\mathbf{x}) \equiv \sum_{n=0}^{\infty} \underbrace{\left(\sum_{m=1}^{b^{n}} \xi_{n_{m}}(\mathbf{x})\right)}_{\xi_{n}(\mathbf{x})}
$$

onde $b^{n} \times b^{n}, \operatorname{com} n=0,1,2, \ldots$, é o tamanho de cada elemento, $m=1,2, \ldots, b^{n}$ é o número de malhas em cada nível $n$ e, $\xi_{n_{m}}(\mathbf{x})$ e $\xi_{n}(\mathbf{x})$ são as variáveis aleatórias Gaussianas independentes. Este procedimento numérico têm sido aplicado com êxito na simulação de escoamentos de fluidos em meios porosos $[2,4,5]$.

\section{RESOLUÇÃO NUMÉRICA DO SISTEMA DE EQUAÇÕES DIFERENCIAIS PARCIAIS}

Na resolução do problema de pressão-velocidade (2.2), para as malhas fina e grossa, as equações diferenciais parciais são discretizadas pelo método dos elementos finitos mistos, que são adequados para o cálculo acurado dos fluxos nos campos de permeabilidades heterogêneos [1]. O sistema algébrico linear associado ao campo de pressões foi resolvido por um método iterativo do tipo Gradiente Conjugado com Pré-condicionador [8, 7].

Na resolução da equação de advecção que governa o transporte do traçador (2.1) emprega-se o método lagrangeano localmente conservativo Forward Integral Tracking (FIT) [2]. Este método prescinde da solução de problemas de Riemann e utiliza a ideia dos tubos integrais introduzida em [11]. Além disso, o FIT é teoricamente livre de difusão numérica e possui uma excelente eficiência computacional.

Os mesmos métodos numéricos utilizados na resolução das equações diferenciais (2.1) e (2.2) são considerados na resolução do transporte advectivo linear da concentração do traçador na malha grossa (2.7) e do problema elíptico (2.8).

\section{A ABORDAGEM BAYESIANA}

Neste trabalho, as simulações de quantificação de incertezas são empregadas com o intuito de atualizar os campos de permeabilidades $k(\mathbf{x})$, baseado-se no conhecimento prévio de um conjunto de valores medidos da concentração do traçador em alguns pontos específicos do meio poroso $O\left(c_{o}\right)$, tal que,

$$
O\left(c_{o}\right)=\left\{c_{o}\left(\mathbf{x}_{i}, t_{j}\right) \mid i=1, \ldots, N_{s} ; j=1, \ldots, N_{t}\right\},
$$

onde $c_{o}\left(\mathbf{x}_{i}, t_{j}\right)$ é a concentração observada do traçador no sensor localizado em $\mathbf{x}_{i}$ de $\Omega$ para o instante de tempo $t_{j}, N_{s}$ é o número de sensores instalados em $\Omega$ e $N_{t}$ representa o número de 
vezes que a concentração é avaliada no tempo. Para se obter as informações referentes aos elementos do conjunto $O\left(c_{o}\right)$, gera-se, inicialmente, um campo de permeabilidades de referência $k_{r}(\mathbf{x})$, onde assume-se que os valores das permeabilidades nos pontos $\mathbf{x}_{i}$ são conhecidos. Posteriormente, resolve-se acuradamente o modelo matemático (2.1)-(2.2) a fim de determinar-se os valores observados da concentração do traçador $c_{o}\left(\mathbf{x}_{i}, t_{j}\right)$, em uma malha fina.

As informações referentes aos valores observados da concentração são introduzidos no modelo através da função de verossimilhança $P\left(O\left(c_{o}\right) \mid k(\mathbf{x})\right)$. Esta informação adicional é utilizada na seleção dos novos campos de permeabilidades através do Teorema de Bayes [10]

$$
\pi(k(\mathbf{x})) \propto P\left(O\left(c_{o}\right) \mid k(\mathbf{x})\right) P(k(\mathbf{x})),
$$

onde $P(k(\mathbf{x}))$ e $\pi(k(\mathbf{x}))=P\left(k(\mathbf{x}) \mid O\left(c_{o}\right)\right)$ são, respectivamente, as distribuições de probabilidade a priori e a posteriori do campo de permeabilidades $k(\mathbf{x})$. A distribuição a priori é dada por [20]

$$
P\left(\xi_{n_{m}}\right)=\exp \left(-\frac{\left\|\xi_{n_{m}}-\xi_{n_{m 0}}\right\|^{2}}{2 \sigma_{\xi_{n_{m}}}^{2}}\right),
$$

onde $\xi_{n_{m 0}}=0$ e $\sigma_{\xi_{n m}}^{2}=1$. Supõe-se que a função de verossimilhança segue uma distribuição normal [10], isto é,

$$
P\left(O\left(c_{o}\right) \mid k(\mathbf{x})\right) \propto \exp \left\{-\frac{\sum_{j=1}^{N_{t}} \sum_{i=1}^{N_{s}}\left[c_{s}\left(\mathbf{x}_{i}, t_{j}\right)-c_{o}\left(\mathbf{x}_{i}, t_{j}\right)\right]^{2}}{2 \sigma_{f}^{2}}\right\},
$$

onde $c_{s}\left(\mathbf{x}_{i}, t_{j}\right)$ é a concentração simulada do traçador no sensor localizado em $\mathbf{x}_{i}$ para o instante de tempo $t_{j}$ obtida com os campos de permeabilidades propostos. Para cada amostra proposta do campo de permeabilidades $k(\mathbf{x})$, obtém-se a concentração simulada do traçador $c_{s}\left(\mathbf{x}_{i}, t_{j}\right)$ a partir da solução numérica do modelo matemático (2.1)-(2.2) na escala fina. O parâmetro $\sigma_{f}^{2}$ é a precisão associada às medidas simuladas e observadas. Assim, o erro em malha fina é definido por [10]

$$
\mathcal{E}_{f}=\sum_{j=1}^{N_{t}} \sum_{i=1}^{N_{s}}\left[c_{s}\left(\mathbf{x}_{i}, t_{j}\right)-c_{o}\left(\mathbf{x}_{i}, t_{j}\right)\right]^{2}=\left\|c_{s}\left(\mathbf{x}_{i}, t_{j}\right)-c_{o}\left(\mathbf{x}_{i}, t_{j}\right)\right\|^{2},
$$

onde $\|\bullet\|$ é a norma $L_{2}$.

Para o modelo matemático na escala grossa, define-se a função de verossimilhança como sendo dada por

$$
\bar{P}\left(O\left(c_{o}\right) \mid k(\mathbf{x})\right) \propto \exp \left(-\frac{\sum_{j=1}^{N_{t}} \sum_{i=1}^{N_{s}}\left(\bar{c}_{s}\left(\mathbf{x}_{i}, t_{j}\right)-c_{o}\left(\mathbf{x}_{i}, t_{j}\right)\right)^{2}}{2 \sigma_{g}^{2}}\right),
$$

onde $\bar{c}_{s}\left(\mathbf{x}_{i}, t_{j}\right)$ é a concentração simulada do traçador obtida a partir da resolução numérica do modelo matemático na escala de campo (2.7) - (2.8), e $\sigma_{g}^{2}$ é a precisão associada ao modelo na escala grossa. Neste caso, define-se o erro em malha grossa como

$$
\mathcal{E}_{g}=\sum_{j=1}^{N_{t}} \sum_{i=1}^{N_{s}}\left(\bar{c}_{s}\left(\mathbf{x}_{i}, t_{j}\right)-c_{o}\left(\mathbf{x}_{i}, t_{j}\right)\right)^{2}=\left\|\bar{c}_{s}\left(\mathbf{x}_{i}, t_{j}\right)-c_{o}\left(\mathbf{x}_{i}, t_{j}\right)\right\|^{2} .
$$


Desta forma, a distribuição de interesse na malha grossa é aproximada por

$$
\bar{\pi}(k(\mathbf{x})) \propto \bar{P}\left(O\left(c_{o}\right) \mid k(\mathbf{x})\right) P(k(\mathbf{x})) .
$$

\section{O MÉTODO DE MONTE CARLO VIA CADEIAS DE MARKOV}

A ideia básica dos métodos MCMC é simular Cadeias de Markov ergódicas $\left\{k^{(\mathrm{t})}\right\}$ que têm como sua distribuição estacionária (ou de equilíbrio) a distribuição a posteriori de interesse $\pi(k(\mathbf{x}))$. Para a construção destas Cadeias de Markov, os métodos MCMC fazem uso de algoritmos específicos, nos quais pode-se destacar o algoritmo de Metropolis-Hastings [10, 14, 16]. Para cada iteração, um campo de permeabilidades $k(\mathbf{x})$ é proposto usando uma distribuição instrumental $q\left(k \mid k^{(\mathrm{t})}\right)$, onde o instante $\mathrm{t}$ representa o estado atual em que se encontra a cadeia de Markov. Em seguida, resolve-se o sistema de equações diferenciais parciais (2.1)-(2.2). Então, o candidato $k$ (x) será aceito como o próximo estado da cadeia com probabilidade dada por

$$
\alpha\left(k \mid k^{(\mathrm{t})}\right)=\left\{\begin{array}{cl}
\min \left(\frac{\pi(k) q\left(k^{(\mathrm{t})} \mid k\right)}{\pi\left(k^{(\mathrm{t})}\right) q\left(k \mid k^{(\mathrm{t})}\right)}, 1\right) & , \quad \pi\left(k^{(\mathrm{t})}\right) q\left(k \mid k^{(\mathrm{t})}\right)>0, \\
1 & , \quad \text { caso contrário, }
\end{array}\right.
$$

tal que $k^{(\mathrm{t}+1)}=k$ com probabilidade $\alpha\left(k \mid k^{(\mathrm{t})}\right)$ e $k^{(\mathrm{t}+1)}=k^{(\mathrm{t})}$ com probabilidade $1-\alpha\left(k \mid k^{(\mathrm{t})}\right)$. Neste trabalho, considera-se o caso especial do algoritmo de Metropolis-Hastings baseado em passeios aleatórios (random walk), onde aplica-se à variável $\xi_{n_{m}}(\mathbf{x})$ ao invés da variável $\xi_{n}(\mathbf{x})$, ou $Y(\mathbf{x})$, a fim de manter-se a mesma estrutura de construção dos campos Gaussianos $Y(\mathbf{x})$ gerados pela técnica SSCGI. Assim, propõe-se $Y(\mathbf{x})$ a partir do novo valor de $\xi_{n_{m}}(\mathbf{x})$ dado por

$$
\xi_{n_{m}}(\mathbf{x})=\sqrt{1-h_{\xi}} \xi_{n_{m}}^{(\mathrm{t})}(\mathbf{x})+\sqrt{h_{\xi}} z \xi
$$

onde $\xi_{n_{m}}^{(\mathrm{t})}(\mathbf{x})$ representa o estado atual da variável aleatória Gaussiana, $n=0,1,2, \ldots$ e $m=1,2, \ldots, b^{n}$. O parâmetro $h_{\xi}$ determina o tamanho do salto em cada passo do algoritmo de Metropolis-Hastings e $z \xi$ é gerado a partir de uma distribuição normal com média 0 e variância 1 , isto é, $z \xi \sim N(0,1)$. O processo de amostragem (6.1) é baseado em passeios aleatórios (random walk) do tipo autoregressivo [16]. A utilização deste tipo de amostragem é bastante vantajosa, pois não se tem o problema de renormalização da variável aleatória $\xi_{n_{m}}(\mathbf{x})$.

Geralmente, as simulações diretas do método MCMC apresentam a desvantagem de necessitar de um elevado esforço computacional, uma vez que um grande número de simulações é necessário para que obtenha-se a convergência da cadeia de Markov e uma boa taxa de aceitação. Portanto, a fim de minimizar este problema recorre-se a uma eficiente e rigorosa metodologia, conhecida como método MCMC a dois estágios [6, 12, 13], que pode ser descrito pelo Algoritmo 1. 


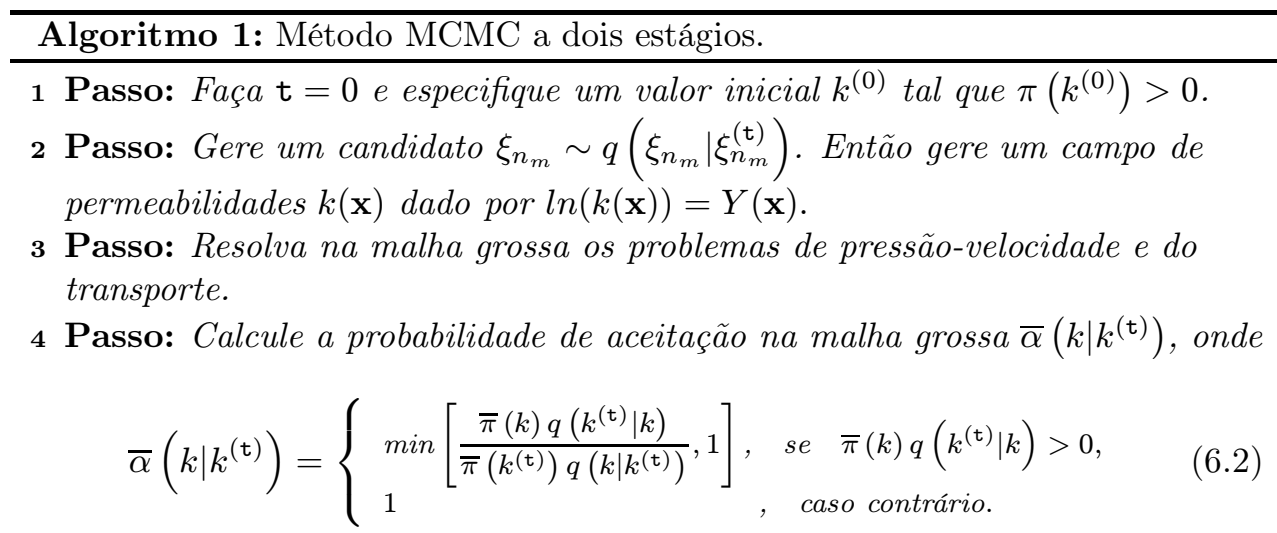

5 Passo: Gere uma variável aleatória u com distribuição uniforme $\mathcal{U}(0,1)$ e teste se o campo proposto será aceito ou não, isto é,

$$
k^{(\mathrm{t}+1)}= \begin{cases}k \quad(\text { condicionalmente }), & \text { se } \mathrm{u} \leq \bar{\alpha}\left(k \mid k^{(\mathrm{t})}\right), \\ k^{(\mathrm{t})} \quad(\text { condicionalmente }), & \text { caso contrário. }\end{cases}
$$

Se o campo proposto $k$ for rejeitado retorne ao Passo 2.

6 Passo: Resolva na malha fina os problemas de pressão-velocidade e do transporte.

7 Passo: Calcule a probabilidade de aceitação na malha fina $\alpha\left(k \mid k^{(\mathrm{t})}\right)$, onde

$$
\alpha\left(k \mid k^{(\mathrm{t})}\right)=\left\{\begin{array}{l}
\min \left[\frac{\pi(k) Q\left(k^{(\mathrm{t})} \mid k\right)}{\pi\left(k^{(\mathrm{t})}\right) Q\left(k \mid k^{(\mathrm{t})}\right)}, 1\right], \\
1 \\
, \quad \text { se } \quad \pi(k) Q\left(k^{(\mathrm{t})} \mid k\right)>0
\end{array}\right.
$$

8 Passo: Gere uma variável aleatória u com distribuição uniforme $\mathcal{U}(0,1)$ e teste se o campo proposto será aceito ou não, isto é,

$$
k^{(\mathrm{t}+1)}= \begin{cases}k, & \text { se } \mathrm{u} \leq \alpha\left(k \mid k^{(\mathrm{t})}\right), \\ k^{(\mathrm{t})}, & \text { caso contrário. }\end{cases}
$$

Se o campo proposto $k$ for rejeitado, retorne ao Passo 2.

9 Passo: Incremente $\mathrm{t}=\mathrm{t}+1$, retorne ao Passo 2 e continue o procedimento até atingir a convergência. 


\section{RESULTADOS NUMÉRICOS}

As simulações foram realizadas com um código numérico serial considerando um tempo máximo de simulação de até 1.700 dias, em uma região física bidimensional com dimensões $L_{x}=128 \mathrm{~m}$ e $L_{y}=128 \mathrm{~m}$. As simulações foram feitas empregando-se diferentes malhas na escala grossa: $16 \times 16,32 \times 32$ e $64 \times 64$ elementos. A malha usada na escala fina foi mantida fixa e possui $128 \times 128$ elementos. O passo de tempo utilizado na resolução da equação de transporte linear foi de $\Delta t=1$ dia. Em todas as simulações o traçador é injetado uniformemente a uma taxa constante de 0,2 volumes porosos a cada ano. O número inicial de pontos sobre a frente de avanço do traçador é de 256 pontos. Para evitar a influência da injeção nos resultados numéricos, no instante inicial especifica-se que o salto esteja localizado a $8 \mathrm{~m}$ de distância da fronteira esquerda do reservatório. Na caracterização da rocha do reservatório utiliza-se campos Gaussianos aleatórios obtidos com covariância espacial fractal. O expoente de Hurst $(\beta)$ é igual a 0,5 e o fator que controla o grau de heterogeneidade do meio foi fixado em 0,4 .

Para a obtenção dos resultados numéricos foram propostas 70.000 amostras, nas quais selecionou-se conjuntos de realizações com 1.000 amostras aceitas de campos de permeabilidades. $\mathrm{O}$ parâmetro de perturbação $h_{\xi}$ do processo de amostragem do tipo random walk foi igual a 0,0025 e o valor da precisão da medida observada foi $\sigma_{g}^{2}=4 \sigma_{f}^{2}$, com $\sigma_{f}^{2}=1,0$. As localizações dos sensores $S_{i}=S_{i}(x, y), i=1, \ldots, 6$, de monitoramento são: $S_{1}=(35,0,32,0), S_{2}=(35,0,64,0)$, $S_{3}=(35,0,96,0), S_{4}=(75,0,32,0), S_{5}=(75,0,64,0)$ e $S_{6}=(75,0,96,0)$ (veja a Figura 3). O valor prescrito da permeabilidade em cada sensor é $k_{0} \neq 0$ e a concentração $c\left(\mathbf{x}_{i}, t_{j}\right)$ foi avaliada a cada 1 dia de simulação.

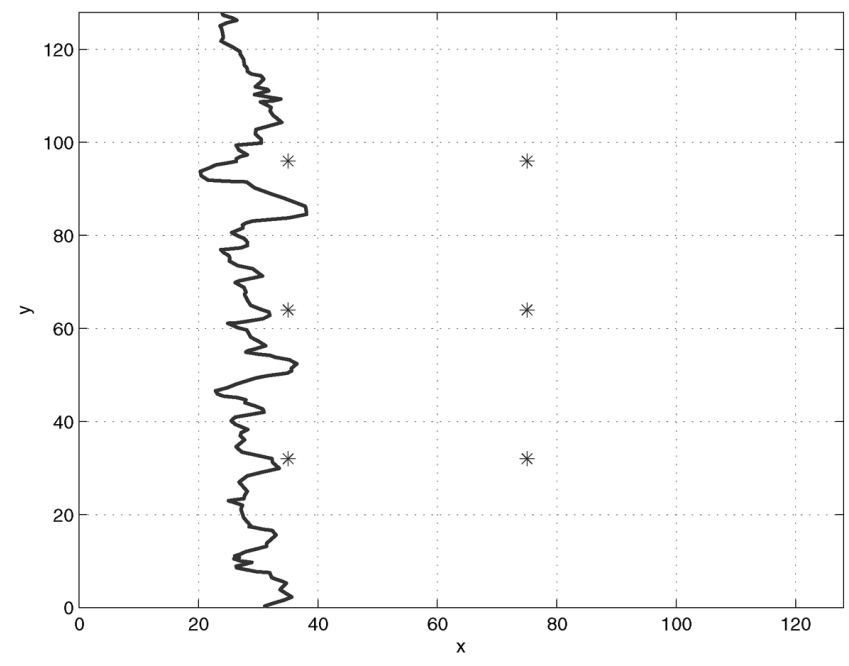

Figura 3: Frente de avanço do traçador e sensores de monitoramento.

Na Tabela 1 são apresentados as taxas de aceitação, para as 1.000 primeiras amostras aceitas de campos de permeabilidades, obtidas com as malhas grossas de $16 \times 16,32 \times 32$ e $64 \times$ 64 elementos. Observa-se que quando passa-se de uma malha de $16 \times 16$ elementos para uma 
malha de $32 \times 32$ e $64 \times 64$ a taxa de aceitação reduz 3, 2\% e 2, 1\%, respectivamente. Esperase que quanto mais próxima a malha grossa está da malha fina os valores da taxa de aceitação aumentariam, uma vez que se elas fossem iguais os mesmos campos de permeabilidades seriam aceitos nos testes de aceitação do método MCMC a dois estágios. Entretanto, deve-se levar em consideração que estas variações dependem das relações entre os erros em malha grossa e fina e, principalmente, dos valores das precisões das medidas, $\sigma_{f}^{2}$ e $\sigma_{g}^{2}$. Além disso, um outro fator que também influência na taxa de aceitação é o refinamento das malhas utilizadas na escala grossa.

Tabela 1: Taxas de aceitação para 1.000 primeiras amostras aceitas.

\begin{tabular}{c|c|c|c}
\hline \multicolumn{4}{c}{ Método MCMC a dois estágios } \\
\hline Malhas na escala grossa & $16 \times 16$ & $32 \times 32$ & $64 \times 64$ \\
\hline Propostas & 49.430 & 68.002 & 55.135 \\
\hline Aceitas: malha grossa & 10.738 & 16.365 & 13.861 \\
\hline Aceitas: malha fina & 1.000 & 1.000 & 1.000 \\
\hline Taxa de aceitação & $9,31 \%$ & $6,11 \%$ & $7,21 \%$ \\
\hline
\end{tabular}

Na Figura 4 apresenta-se os campos aleatórios Gaussianos de referência, inicial e outros quatro campos aceitos, os de números 40.344, 43.772, 48.880 e 49.818, dentre os 1.000 primeiros campos aceitos na malha fina com $128 \times 128$ elementos e malhas na escala grossa com $16 \times 16$ elementos. Pode-se observar que os campos aceitos são distintos entre si e que, existe uma diferença significativa entre os campos aleatórios Gaussianos selecionados e os campos de referência e inicial. Portando, conclui-se que o parâmetro de perturbação $h_{\xi}=0,0025$ utilizado no processo de amostragem, para as malhas e a relação entre as precisões das medidas, foi capaz de selecionar campos aleatórios Gaussianos distintos e que incorporam as informações disponíveis a priori.

Com o objetivo de diagnosticar a convergência numérica das cadeias de Markov, adota-se como método a inspeção visual dos erros na malha fina $\mathcal{E}_{f}$ [20]. A Figura 5 apresenta a variação do erro na malha fina em função dos primeiros 1.000 campos de permeabilidades aceitos, obtidos com malhas na escala grossa de $16 \times 16,32 \times 32$ e $64 \times 64$ elementos. Pode-se observar que para todas as malhas as cadeias de Markov convergiram para a distribuição estacionária de interesse $\pi(k(\mathbf{x}))$. Os erros decresceram de valores da ordem de aproximadamente $5 \cdot 10^{2}$ para $10 \cdot 10^{0}$. Os valores baixos destes erros são a garantia de que os campos aceitos possam fornecer, para a concentração simulada do traçador $c_{s}\left(\mathbf{x}_{i}, t_{j}\right)$, valores próximos aos medidos nos sensores $c_{o}\left(\mathbf{x}_{i}, t_{j}\right)$. Esse fato pode ser verificado observando-se a Figura 6, que contém os gráficos dos perfis médios de concentração simulada do traçador nas posições correspondentes à localização dos diferentes sensores instalados no meio poroso. Nesta figura, compara-se os perfis oriundos das simulações fazendo o uso dos campos de permeabilidades de referência e inicial, além dos perfis médios da concentração do traçador obtidos para um conjunto de 800 amostras aceitas dos campos de permeabilidades, após o período de aquecimento de 200 campos aceitos. Como se pode observar, em todos os casos, existe uma boa concordância entre os perfis da concentração média simulada do traçador e os observados. 


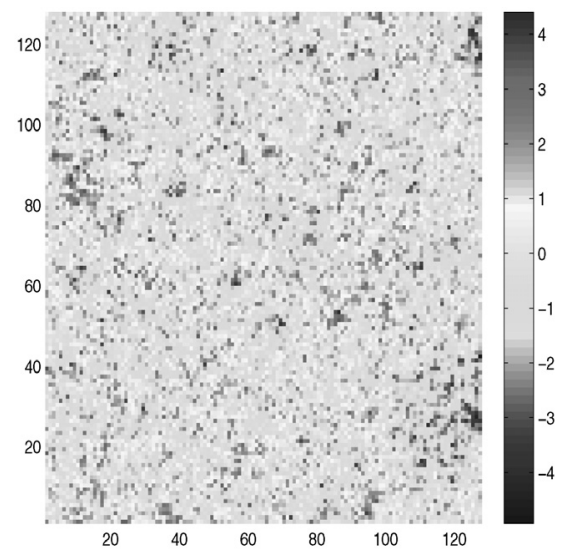

(a) Campo de referência.

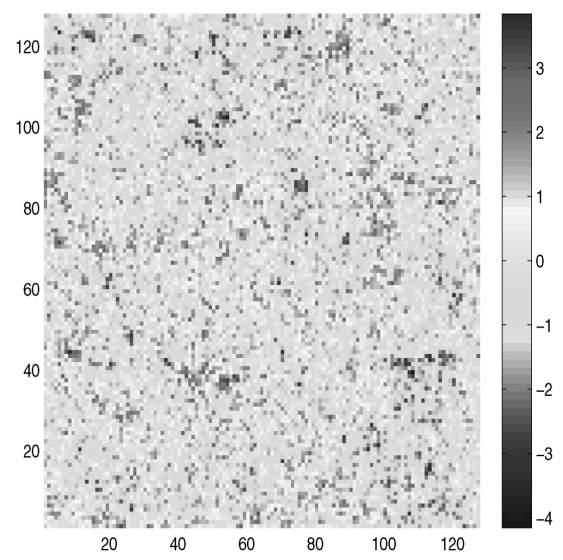

(c) Campo aceito $n^{\circ} 40.344$

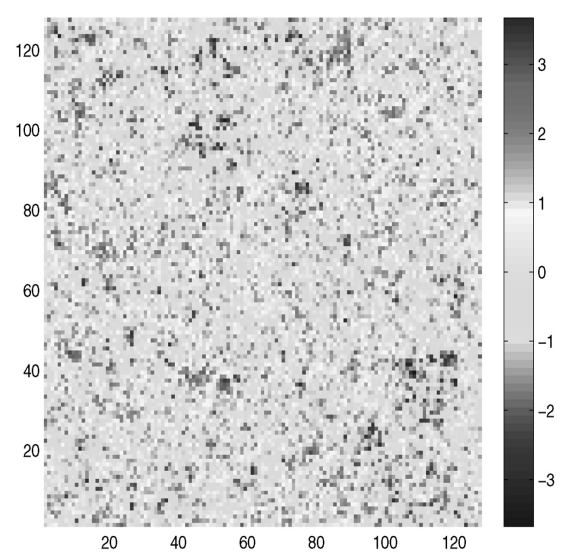

(e) Campo aceito $\mathrm{n}^{\circ} 48.880$.

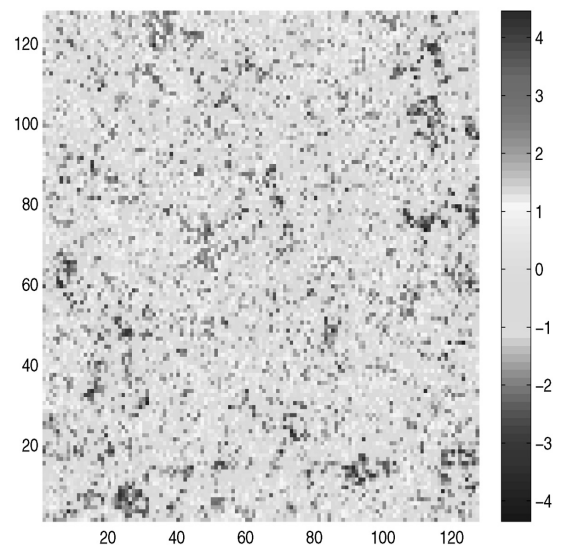

(b) Campo inicial.

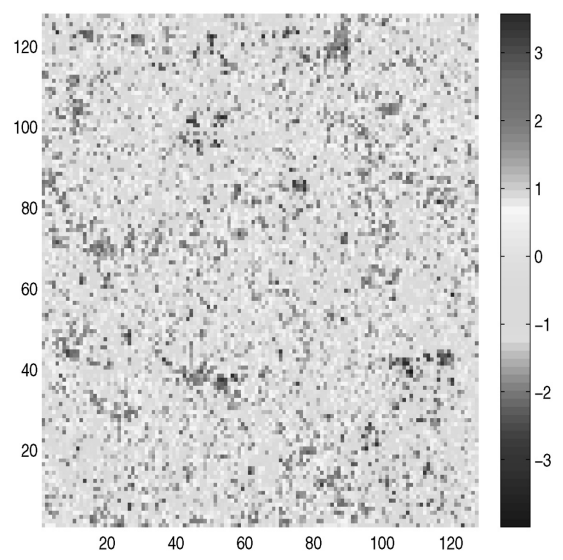

(d) Campo aceito ${ }^{\circ} 43.772$.

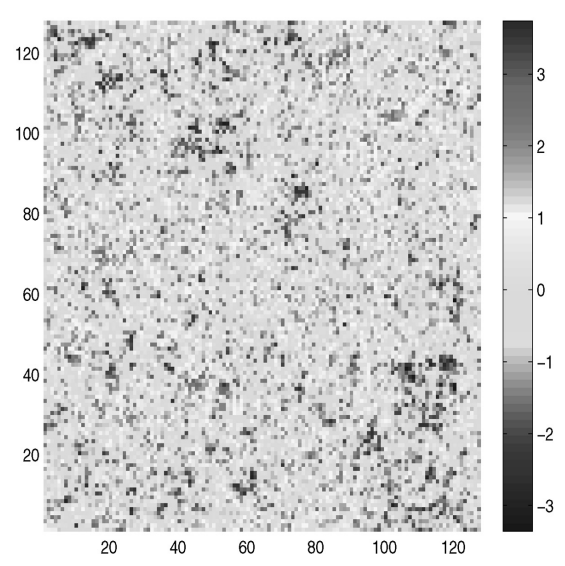

(f) Campo aceito $n^{\circ} 49.818$.

Figura 4: Realizações aceitas dos campos aleatórios Gaussianos. 
A partir dos resultados numéricos obtidos, entende-se que esta abordagem foi capaz de propor a atualização dos campos de permeabilidades e reduzir as incertezas contidas no modelo matemático que descreve o transporte de um traçador.

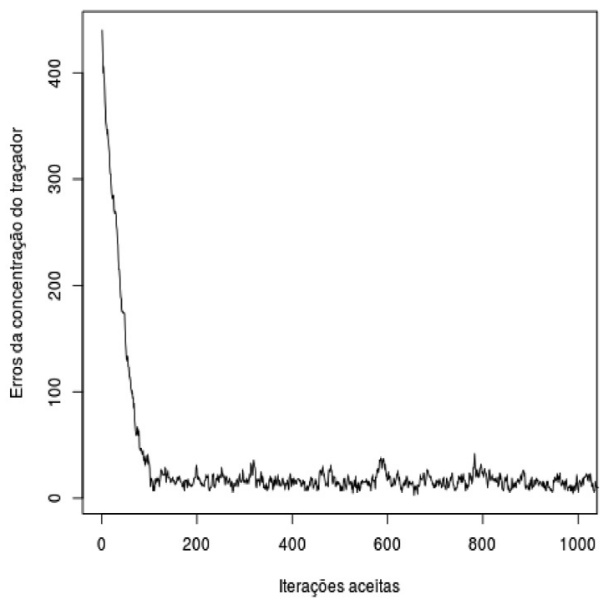

(a) Malhas de $16 \times 16$ elementos.

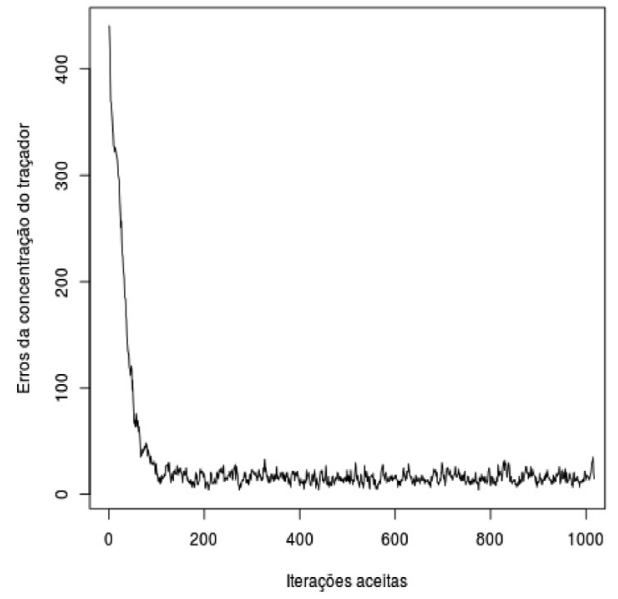

(b) Malhas de $32 \times 32$ elementos.

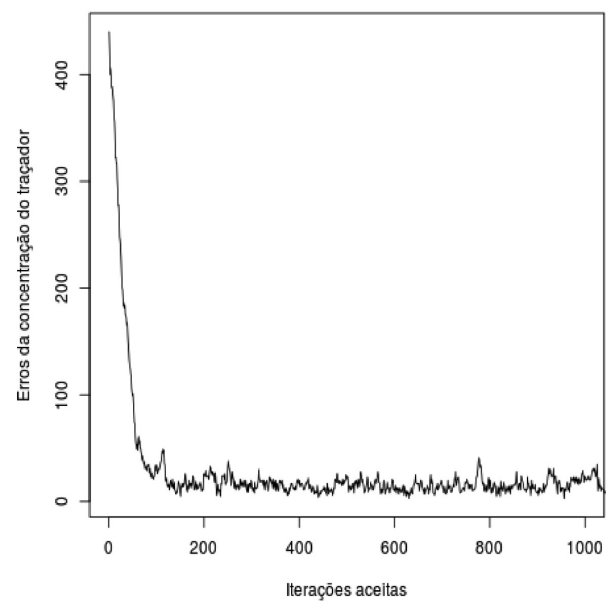

(c) Malhas de $64 \times 64$ elementos.

Figura 5: Erro na malha fina da concentração do traçador em função das amostras aceitas.

\section{CONCLUSÃO}

Neste trabalho foi utilizado uma abordagem Bayesiana para a atualização dos campos de permeabilidades, baseada no conhecimento prévio dos valores da concentração do traçador e da permeabilidade em alguns pontos específicos do meio poroso heterogêneos. O método da Soma Sucessiva de Campos Gaussianos Independentes foi usado para gerar as amostras dos campos de permeabilidades na solução do problema inverso estocástico associado ao escoamento do 


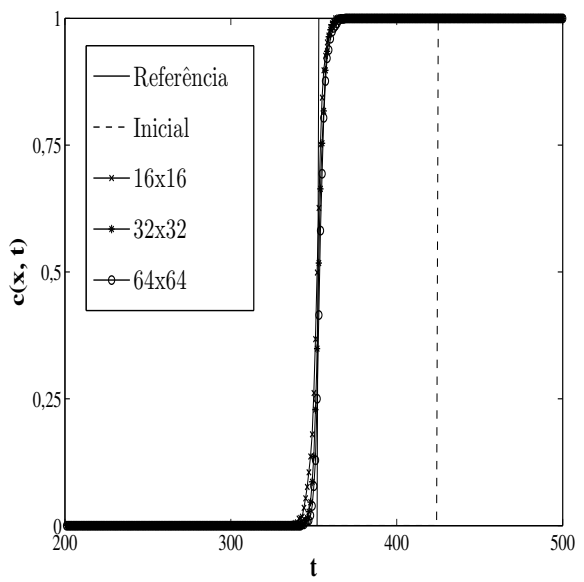

(a) Sensor 0 .

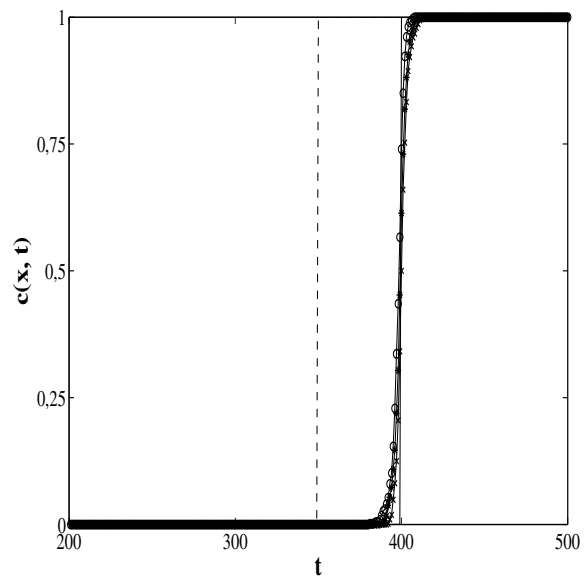

(c) Sensor 2 .

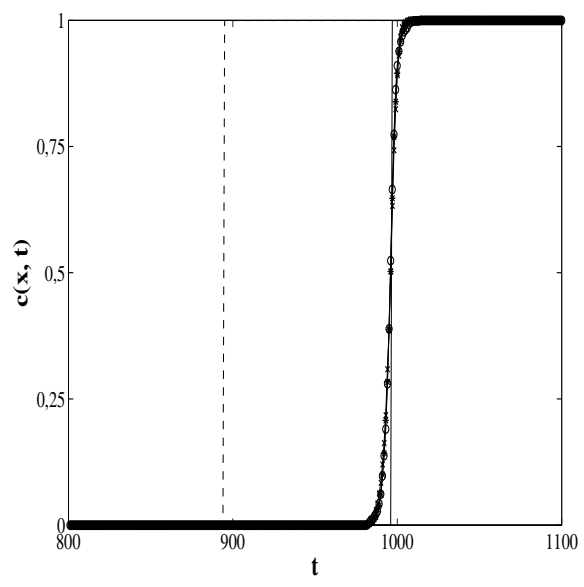

(e) Sensor 4 .

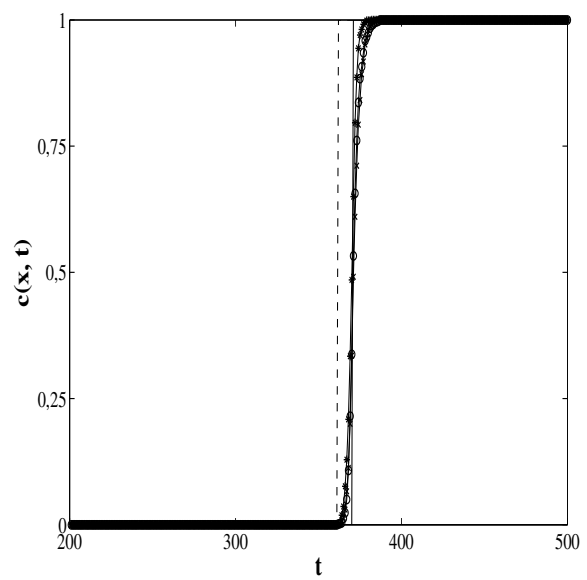

(b) Sensor 1 .

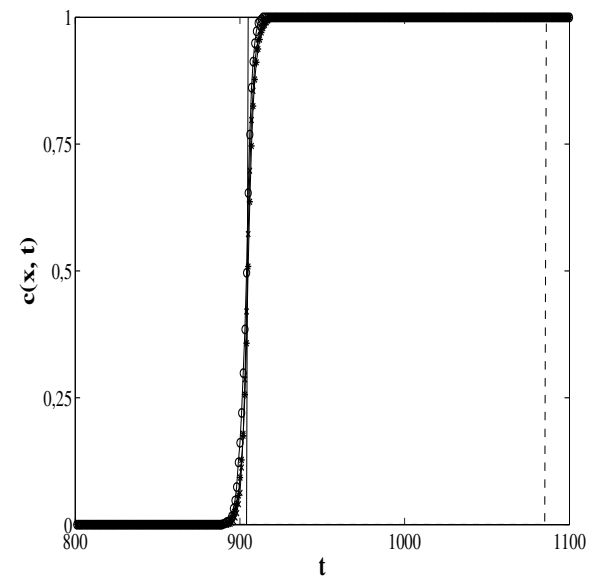

(d) Sensor 3 .

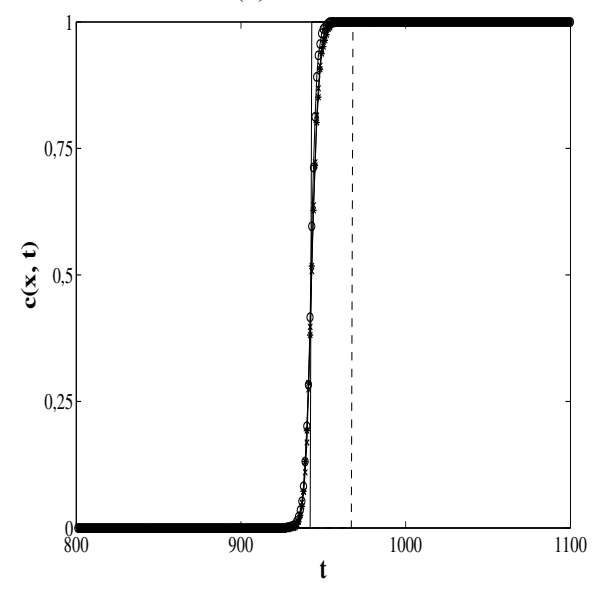

(f) Sensor 5 .

Figura 6: Valores da concentração do traçador em diferentes sensores. 
traçador em formações heterogêneas. Na solução do problema inverso complexo, foi utilizado o método Monte Carlo via Cadeias de Markov a dois estágios, cujo o processo de amostragem é do tipo random walk onde emprega-se um modelo autoregressivo. Os resultados numéricos obtidos indicam que as cadeias de Markov convergiram para a distribuição a posteriori de interesse, o parâmetro $h_{\xi}=0,0025$ usado no processo de amostragem do modelo autoregressivo seleciona campos de permeabilidades distintos entre si e as incertezas contidas no modelo matemático que descreve o escoamento de um traçador no interior de um meio poroso podem ser reduzidas a partir do conhecimento prévio das medições dos valores das concentrações do traçador em alguns pontos específicos do meio poroso. Como perspectiva futura do presente trabalho cita-se, por exemplo, a utilização do método MCMC a dois estágios na quantificação de incertezas de problemas envolvendo escoamentos multifásicos.

\begin{abstract}
The goal of this work is to study the uncertainty quantification in inverse problems of miscible flows in heterogeneous porous media in Bayesian framework and propose a permeability update based on observed measurements of spatially sparse tracer concentration at certain times. The Successive Sum of Independent Gaussian Fields method is used to parametrization of the uncertainty in heterogeneous porous media. A two-stage Markov chain Monte Carlo (MCMC) method is used to solving the inverse problem. For the construction of Markov chains is used the Metropolis-Hastings algorithm based on a random walk sampling process employs an auto-regressive instrumental distribution. The main idea of MCMC is to generate a Markov chain with an equilibrium distribution equal to the posterior distribution of interest. Numerical results are presented for a set sampled realizations of the permeability fields.
\end{abstract}

Keywords: Gaussian stochastic field, porous media flow, two-stage MCMC method, tracer.

\title{
REFERÊNCIAS
}

[1] J. Aquino, A.S. Francisco, F. Pereira \& H.P. Amaral Souto. Numerical simulation of transient water infiltration in heterogeneous soils combining central schemes and mixed finite elements. Communications in Numerical Methods in Eng., 23 (2007), 491-505.

[2] J. Aquino, A.S. Francisco, F. Pereira \& H.P. Amaral Souto. A hybrid method for the simulation of radionuclide contaminant plumes in heterogeneous unsaturated formations. Progress in Nuclear Energy, 53 (2011), 1159-1166.

[3] G.R. Barth, T.H. Illangasekare, M.C. Hill \& H. Rajaram. A New Tracer-density Criterion for Heterogeneous Porous Media. Water Resources Research, 37(1) (2001), 21-31.

[4] M.R. Borges, F. Pereira, F. Furtado \& H.P. Amaral Souto. Scaling analysis for the tracer flow problem in self-similar permeability fields. SIAM Multiscale Modeling and Simulation, 7(3) (2008), 11301147.

[5] M.R. Borges, F. Pereira \& H.P. Amaral Souto. Efficient generation of multi-scale random fields: A hierarchical approach. International Journal for Numerical Methods in Biomedical Engineering, 26 (2010), 176-189. 
[6] J.A. Chrsitien \& C. Fox. Markov chain Monte Carlo using an approximation. Journal of Computational and Graphical Statistics, 14(4) (2005), 795-810.

[7] M.C.C. Cunha. Métodos numéricos. Journal of Computational and Graphical Statistics, 14(4) (2005), 795-810.

[8] M.C.C. Cunha. "Métodos numéricos". Editora da UNICAMP, Campinas (2000).

[9] L.J. Durlofsky. Coarse Scale Models of two Phase in Heterogenous Reservoirs: Volume Averaged Equations and Their Relationship to the Existing Upscaling Techniques. Computational Geosciences, 2(2) (1998), 73-92.

[10] C. Douglas, Y. Efendiev, R. Ewing, V. Ginting \& R. Lazarov. Dynamic Data Drive Simulations in Stochastic Environments. Computing, 77 (2006), 321-333.

[11] J. Douglas Jr, F. Pereira \& L.M. Yeh. A Locally Conservative Eulerian-Lagrangian Numerical Method its Aplication to nonlinear transport in Porous Media. Computational Geosciences, 4 (2000), 1-40.

[12] Y. Efendiev, A. Datta-Gupta, V. Ginting, X. Ma \& B. Mallick. An Efficient Two-Stage Markov Chain Monte Carlo Method for Dynamic Data Integration. Water Resources Research, 41(12) (2005).

[13] Y. Efendiev, T. Hou \& W. Lou. Proconditioning Markov Chain Monte Carlo Simulations Using Coarse-Scale Models. SIAM J. Sci. Comput, 2(2) (2006), 776-803.

[14] Y. Efendiev, A. Millick \& A. Datta-Gupta. Bayesian Uncertainty Quantification for Flows in Heterogeneus Porous Media Using Reversible Jump Markov Chain Monte Carlo Methods. Advances in Water Resources, 33(3) (2010), 241-256.

[15] P. Engesgaard, K.H. Jensen, J. Molson, E.O. Frind \& H. Olsen. Large-scale Dispersion in a Sandy Aquifer: Simulation of Subsurface Transport of Envionmental Tritium. Water Resources Research, 32(11) (1996), 3253-3266.

[16] D. Gamerman \& H.F. Lopes. "Markov Chain Monte Carlo: Stochastic Simulation for Bayesian Inference”. Texts in Statistical Science, Second Edition, Chapman \& Hall/CRC (2006).

[17] K.H. Jensen, K. Bitsch \& P.L. Bjerd. Large-scale dispersion experiments in a sandy aquifer in Denmark: Observed tracer movements and numerical analyses. Water Resources Research, 29(3) (1993), 673-696.

[18] A.F. Loula, J.N.C. Guerreiro, F.L.B. Ribeiro \& L. Landau. Tracer Injection Simulations by Finite Element Methods. Society of Petroleum Engineers, 4(1) (1996), 150-156.

[19] A.F. Loula, E.L.M. Garcia \& A.L.G.A. Coutinho. Miscible Displacement Simulation by Finite Element Method in Distributed Memory Machines. Computer Methods in Applied Mechanics and Engineering, 174 (1999), 339-354.

[20] A. Mondal, Y. Efendiev, A. Millick \& A. Datta-Gupta. Bayesian Uncertainty Quantification for Flows in Heterogeneus Porous Media Using Reversible Jump Markov Chain Monte Carlo Methods. Advances in Water Resources, 33(3) (2010), 241-256.

[21] G. Victor, P. Felipe, P. Michael \& W. Shaochang. Application of the two-stage Markov chain Monte Carlo method for characterization of fractured reservoirs using a surrogate flow model. Computational Geosciences, 15(4) (2011), 691-707.

[22] T. Ptak, M. Piepenbrink \& E. Martac. Tracer tests for the investigation of heterogeneous porous media and stochastic modelling of flow and transport - a review of some recent developments. Journal of Hydrology, 294 (2004), 122-163. 